\title{
EFFICIENT SYSTEM COMBINATION FOR SYLLABLE-CONFUSION-NETWORK-BASED CHINESE SPOKEN TERM DETECTION
}

\author{
Jie Gao, Qingwei Zhao, Yonghong Yan* \\ ThinkIT Speech Lab, Institute of Acoustics, \\ Chinese Academy of Sciences, Beijing \\ \{jgao,qzhao,yanyonghong @hccl.ioa.ac.cn\}
}

Jian Shao

\author{
Zhejiang University \\ Hangzhou, Zhejiang \\ \{jshao@zju.edu.cn\}
}

\begin{abstract}
This paper examines the system combination issue for syllableconfusion-network (SCN) -based Chinese spoken term detection (STD). System combination for STD usually leads to improvements in accuracy but suffers from increased index size or complicated index structure. This paper explores methods for efficient combination of a word-based system and a syllable-based system while keeping the compactness of the indices. First, a composite SCN is generated using two approaches: lattice combination (The SCN is generated from a combined lattice) and confusion network combination (Two SCNs are combined into one). Then a simple compact index is constructed from this composite SCN by merging crosssystem redundant information. The experimental result on a 60 -hour corpus shows a relative accuracy improvement of $14.7 \%$ is achieved over the baseline syllable-based system. Meanwhile, it reduces the index size by $22.3 \%$ compared to the commonly adopted score combination method when achieves comparable accuracy.
\end{abstract}

Index Terms - syllable confusion network, Chinese spoken term detection, system combination, speech indexing

\section{INTRODUCTION}

With the availability of the ever-increasing amount of the speech archives, rapid and intelligent access to large corpora of speech becomes one of the most important challenges of speech technology. Demands emerge from various areas such as information security, media management, and personal entertainment. These necessitate development of techniques such as Spoken Document Retrieval [4] and Spoken Term Detection.

Recently, Spoken Term Detection (STD) evaluation started by NIST [3] aims to find occurrences of a specified term from large heterogenous speech corpora. Putative occurrences are output with ranking scores and hard decisions indicating their correctness. Many technological challenges are presented for STD, such as vocabulary independence, index size and search speed. Investigations on combination of information from word and various subword units for STD are performed with different motivations, addressing the out of vocabulary $(\mathrm{OOV})$ problems $[1,11]$ and/or utilizing complementary information of different systems $[8,9,10,11,12]$.

A comparison of some previous work is given in Table 1 in terms of their difference in the number and characteristic of index tables. Miller et.al [1] proposes to index the word lattices as well as the top

* This work is partially supported by National NSF of China (10574140, 60535030), MOST (973 program,2004CB318106), the National High Technology Research and Development Program of China (863 program, 2006AA010102,2006AA01Z195). phonetic transcripts from a single Speech-To-Text (STT) system. It is proposed in $[8,9,10,12]$ the output of several STT systems are indexed separately and results are obtained from combing the search results from these indices. Word and phonetic unit are fused into a heterogeneous index ${ }^{1}$ containing multiple units in $[8,11]$. Although accuracy improvement is observed from these methods, keeping multiple or a heterogeneous index tables can be problematic. Multiple indices increase storage and need multi-pass search to detect terms. Complicated match algorithms are required to deal with the heterogeneous structure of index table. In the scenarios where the index size and search speed is critical, a single compact and homogenous index is highly desirable for a system combination method.

Chinese syllable has features which are suitable for STD. About 1200 Chinese syllables in total cover all words in Chinese so there is no OOV problem for the syllable-based Chinese STD systems. In addition, syllable is more acoustically stable and makes better subword unit than phonemes. Therefore, Chinese STD systems based on syllable are presented in $[5,10,12]$. This motivates us to combine a word-based system and a syllable-based system to construct a single homogenous index on the syllable level. Redundant information across systems is also merged, resulting in size reduction of the index. Two approaches are presented for this purpose. In addition, pruning is adopted to further reduce the index size.

In Section 2, we recapitulate our SCN-based spoken term detection [5]. Section 3 introduces the proposed methods to efficiently combine word/syllable systems into one single index. Experimental results and evaluation of the proposed approaches are presented in Section 4 . Section 5 finally concludes this work.

\begin{tabular}{c||l|l|l}
\hline Related work & {$[1,8,9,10,12]$} & {$[8,11]$} & This work \\
\hline \hline Number & $\geq 2$ & 1 & 1 \\
\hline Characteristics & Homo. & Heter. & Homo. \\
\hline
\end{tabular}

Table 1. Taxonomy of some combination work in terms of the number and characteristics of the indices: (Heter:heterogenous, containing different units, Homo:homogeous, containing single unit)

\section{SYLLABLE CONFUSION-NETWORK-BASED STD}

This section briefly recapitulates our SCN-based STD system [5]. Our system consists of two major steps: indexing and searching.

\footnotetext{
${ }^{1}$ If a index table contains multiple units simultaneously, we denote it a heterogenous index, otherwise a homogenous index in this work
} 
During the indexing step, audio input first goes through the STT system that produces syllable confusion networks (SCNs). Then SCNs are indexed into compact searchable database using inverted index technique for information retrieval. For each syllable hypothesis, a soft hit of \{position, confidence, start time, endtime $\}$ is indexed. In retrieval time, a query is represented as a sequence of syllables. Putative occurrences are obtained by merging posting lists of each syllable according to position proximity in the original confusion network. The ranking scores are determined as the accumulated confidence of the soft hits on the matched path.

One fact needs to be pointed out is that SCNs can be generated from STT systems using word or syllable as their decoding unit [5]. Conversion from syllable lattices to SCNs is straightforward [7] for the syllable-based system. For the word-based system, the generation of SCNs is implemented with two steps. First, word lattices are decomposed into syllable lattices using a method similar to these in [10, 12]: a word arc $a$ is split into a sequence of syllable arcs whose posterior probability are assigned as that of $a$. Duration of new created arcs are determined according to phoneme level trace information recorded in the decoding process. Second, syllable lattices from decomposition are decoded into SCNs.

\section{EFFICIENT COMBINATION OF WORD/SYLLABLE SYSTEMS}

Combination of word and syllable systems is proposed in $[5,10,12]$ for Chinese speech-related information retrieval application. However, these methods are inefficient due to the existence of redundancy: the occurrences of the same spoken term exist in the output of both systems, which are unnecessary for the STD task. Base on the fact that syllable is an effective unit for Chinese STD [5, 10, 12], we propose to fuse the output of word/syllable decoding systems into one single syllable-level index and merge the duplication. As show in Figure 1, our starting point is two syllable lattices $L_{1}$ and $L_{2}$ of a same utterance. One is directly derived from the syllable system, and the other is converted from a word lattice following the method described in Section 2. Redundant information are in the form of temporally overlapped lattices arcs corresponding to the same syllable instances.

Two methods are proposed to merge duplicated arcs between the two lattices and generate a composite SCN. In first method (Lat.C), a combined lattice is generated and the composite $\mathrm{SCN}$ is transformed from this lattice. The second method (CN.C) obtains two SCNs separately. Then the two SCNs are combined using confusion network combination [2] technique.

\subsection{Lattice combination}

Lattice union operation is performed on two syllable lattices to obtain a combined lattice [8]. A new start node and a new end node are created. The start node and the end node of the two lattices are

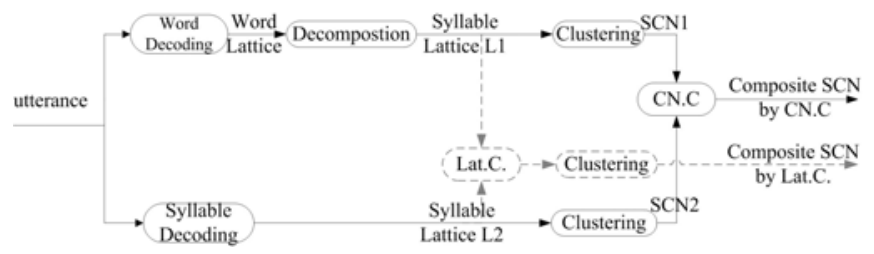

Fig. 1. SCN-based system combination overview

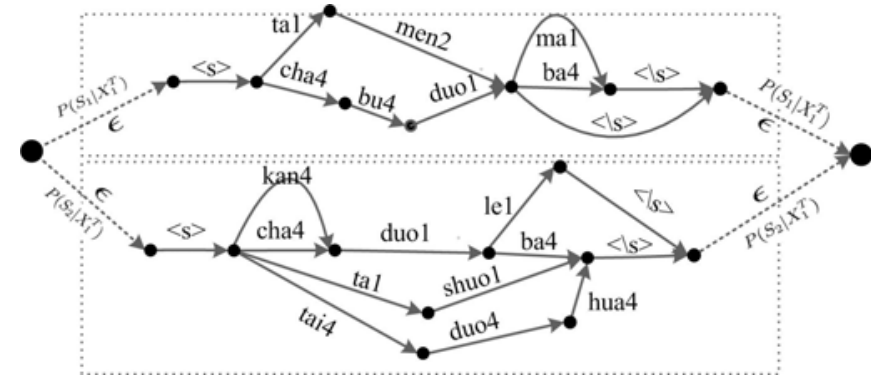

(a) Combining two syllable lattices by lattice union, $\epsilon$ arcs are created for connection

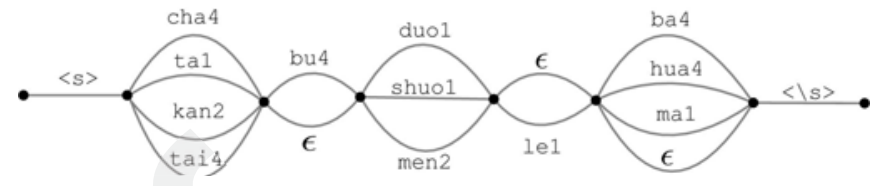

(b) The composite SCN generated from the combined lattice

Fig. 2. Lattice union and corresponding composite SCN

connected to the new start node and end node respectively by $\epsilon$ arcs as shown in Figure 2(a). Then the combined lattice is decoded into a compact SCN.

Fusing two lattices into a single structure helps to merge duplicated lattice arcs. It makes possible that intra-word clustering of the subsequent SCN generation [7] is simultaneously operated on two lattices. Hence the arcs corresponding to the same syllable can be grouped and merged. As shown in Figure 2(b), a compact SCN is finally generated.

A concern for this method is probability mismatch of the two lattices. Because they are generated from different STT systems, their total likelihood may be not of the same scale(one may be 1000 times bigger larger than the other in our observation.) Hence, a probability normalization must be performed to guarantee that probabilities of the two lattices are of the same scale. In this work, as a normalization step, the posterior probability of each arc $a$ in the combined lattice is assigned the value of the weighted posterior probability in the original lattice that it belongs to. It can be obtained by summing up all paths $q$ through this $\operatorname{arc}$ (set $Q_{a}$ ) [2]. Formally,

$$
\begin{aligned}
P_{\text {norm }}\left(a \mid X_{1}^{T}\right) & =P\left(S_{i} \mid X_{1}^{T}\right) \cdot P\left(a \mid X_{1}^{T}, S_{i}\right) \\
& =P\left(S_{i} \mid X_{1}^{T}\right) \cdot \frac{\sum_{q \in Q_{a}} P\left(q, X_{1}^{T}, S_{i}\right)}{P\left(X_{1}^{T}, S_{i}\right)}
\end{aligned}
$$

where $S_{i}$ is the $i$ th system that generates $a$ and $X_{1}^{T}$ is observation, $P\left(S_{i} \mid X_{1}^{T}\right)$ is the system weight determined empirically in this work. This operation is performed before the lattices are combined into a single structure.

\subsection{Composite SCNs from confusion network combination}

In second approach, the system combination is done after the confusion network generation procedure. When two SCNs are transformed from corresponding syllable lattices, confusion network combination (CN.C) [2] is applied for fusion. By defining local cost function for syllable matching given two confusion sets, CN.C first uses dynamic programming (DP) algorithm to align SCNs; then a composite $\mathrm{SCN}$ is created by fusing them together. However, some 


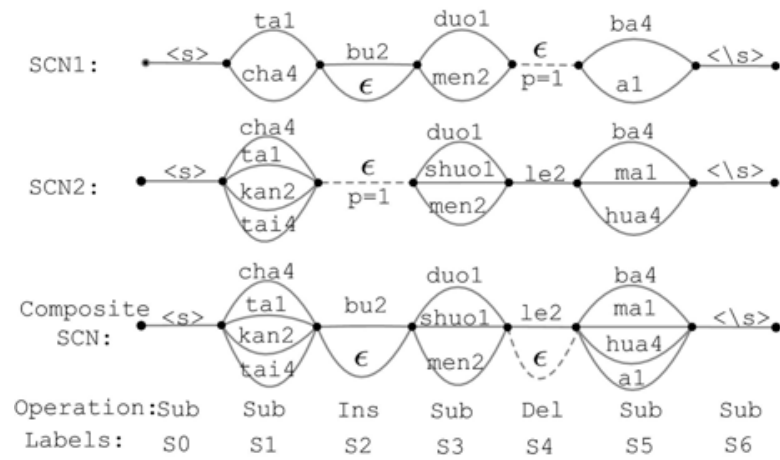

Fig. 3. Alignment of SCNs, composite SCN, operations in DP, and confusion set labels. Arcs in dash line are $\epsilon$ arcs created to represent adjacency in original SCNs. (Ins:insertion, Sub:substitution, Del:deletion )

position proximity information on the original SCNs is lost due to the insertion and deletion operation in the alignment. For example, in Figure 3, the arc labeled with $a 1$ in $S 5$ adjoins $S 3$ in $S C N 1$. But it does not in the composite SCN. Hence, before the fusion step $\epsilon$ arcs with unit weight are created in the positions of substitution and deletion to represent the adjacency relation in the original SCN.An example is shown in Figure 3.

The posterior probability of syllable arc $a$ on the composite $\mathrm{CN}$ given observation $X_{1}^{T}$ is evaluated as weighted sum of the posteriors of two component SCN:

$$
p\left(a \mid X_{1}^{T}\right)=\sum_{i=1}^{2} P\left(S_{i} \mid X_{1}^{T}\right) p\left(a \mid S_{i}, X_{1}^{T}\right)
$$

where $S_{i}$ is the $i$ th system and $P\left(S_{i} \mid X_{1}^{T}\right)$ is the system weight.

CN.C also helps to eliminate part of cross-system redundancy: arcs from two systems with the same syllable label are merged in the positions labeled as substitution by DP. This reduces the index size.

A noticeable point in CN.C is that a posting list is created for all $\epsilon$ arcs in the subsequent indexing stage because our term detection algorithm relies on the adjacency information. This list helps to make the position proximity judgments in the searching stage [5].

\section{EXPERIMENTS}

\subsection{Experimental Setup}

We conduct the STD experiments on a data set that consists of 60.5hour long Chinese conversational telephone speech (CTS) data. It consists of 145 conversational speeches, each of which contains two speakers. A list of 256 terms is selected manually and there are totally 1860 occurrences. The statistics of these terms and their occurrences in number of Chinese syllables are presented in Table 2.

\begin{tabular}{c||c|c|c|c|c|c}
\hline Number of syllables & 2 & 3 & 4 & 5 & $6+$ & total \\
\hline \hline terms & 61 & 122 & 53 & 16 & 4 & 256 \\
\hline occurrences & 631 & 902 & 243 & 70 & 14 & 1860 \\
\hline
\end{tabular}

Table 2. The statistics of the terms
For speech recognition, we use the ThinkIT one-pass recognizer [6]. The acoustic model is trained with about 200 hours of data with 39-dimension features (13-dimension PLP and their first and second order time derivatives). Meanwhile, a dictionary of 44920 words is used for our word decoding system and 1276 tonal Chinese syllables are used for our syllable decoding system. Besides, a word trigram language model is trained with the same data as presented in [5]. A syllable trigram is trained with syllable transcriptions of the corpora used for word language model.

The STD performance is measured in Figure of Merit (FOM). It is defined by National Institute of Science and Technology (NIST) as the recall/false alarms per term per hours. The recall rate (REC), which is an upper bound of FOM, is also listed for analysis purpose.

\subsection{Results}

Experiments are firstly conducted to evaluate the performance of the syllable-based system (Baseline), which serves as the baseline system in this work, and the word-based system (Word) respectively. The results are shown Table 3. It is noticeable that the word system (Word) achieves better performance in all aspects even on OOV words ${ }^{2}$ because OOV words for the word based system can actually backoff onto character level for Chinese [10].

\subsubsection{Accuracy of combination methods}

Two proposed methods as well as the score combination method (Score.C) (weighed combination of scores for putative hits from different systems) $[8,10,12]$ are evaluated. Equal weights are used for all system combination methods. All the methods show performance improvement compared to any single system, as shown in Table 3.

As reported before, the Score.C method shows improvement. The FOM is improved from 58.14 of the best single system to 64.28 . However, it is not efficient in terms of storage and search speed as reported in the next section. The results of the two proposed methods are also shown. The combined lattice method (Lat.C.) and the CNC method (CN.C) achieves FOM of 64.29 and 64.15 respectively, which are comparable to the score combination method. The last row shows the relative improvement of the Lat.C. method compared to the baseline, which achieves the best performance. It is observed an improvement of $31.55 \%$ is achieves on the OOV words and $14.7 \%$ on overall in terms of FOM.

\begin{tabular}{c||c|c||c|c||c|c}
\hline \multicolumn{1}{c||}{} & \multicolumn{2}{c||}{ INV } & \multicolumn{2}{c||}{ OOV } & \multicolumn{2}{c}{ ALL } \\
\hline Systems & REC & FOM & REC & FOM & REC & FOM \\
\hline \hline Baseline & 60.71 & 59.68 & 42.64 & $\mathbf{4 1 . 6 2}$ & 56.88 & $\mathbf{5 6 . 0 5}$ \\
\hline Word & 63.30 & 62.66 & 44.16 & 43.15 & 59.24 & 58.14 \\
\hline Score.C. & 69.03 & 67.81 & 51.26 & 51.24 & 65.27 & 64.28 \\
\hline Lat.C. & 70.40 & 67.73 & 54.82 & $\mathbf{5 4 . 7 5}$ & 65.37 & $\mathbf{6 4 . 2 9}$ \\
\hline CN.C. & 68.55 & 67.17 & 53.04 & 53.02 & 65.27 & 64.15 \\
\hline \hline rel.impr & 15.95 & 13.49 & 28.57 & $\mathbf{3 1 . 5 5}$ & 14.94 & $\mathbf{1 4 . 7 0}$ \\
\hline
\end{tabular}

Table 3. FOM results for all the system combination methods

\subsubsection{Index size and search speed}

Although all the combination method achieves similar accuracy, they are not equally efficient in terms of index and search speed, as shown

\footnotetext{
${ }^{2} \mathrm{OOV}$ words are defined according to the vocabulary of the word system
} 
in Table 4. Search speed is measured in Processing Mil-Second per Term per Hour of Speech (MS.P/HS/T) and index is in Mega Byte per Hour of Speech (MB/HS).

For the Score.C method, the index table size and the search time both increase to sum of those of two component systems because indexing and searching are performed separately. By merging duplications, the Lat.C method reduces the index from $2.66 \mathrm{MB} / \mathrm{HS}$ of the score combination method to $2.06 \mathrm{MB} / \mathrm{HS}$. An index size of $2.13 \mathrm{MB} / \mathrm{HS}$ is also achieved by the CN.C method. This size is slightly bigger than that of the Lat.C method. It can be explained by the fact that merge of CN.C method only occurs among arcs of the same syllable in two aligned confusion sets. But all arcs of the same syllable instances can be merged in the Lat.C method. The relative efficiency of Lat.C to Score.C is shown in the last row, namely $22.31 \%$ size reduction and $16.55 \%$ search time reduction.

\begin{tabular}{c||c|c}
\hline Systems & Index Size(MB/HS) & Speed(MS.P/HS/T) \\
\hline \hline Baseline & 1.56 & 0.67 \\
\hline Word & 1.09 & 0.42 \\
\hline Score.C. & $\mathbf{2 . 6 6}$ & $\mathbf{1 . 1 0}$ \\
\hline Lat.C. & $\mathbf{2 . 0 6}$ & $\mathbf{0 . 9 1}$ \\
\hline CN.C. & 2.13 & 0.97 \\
\hline rel.red & $\mathbf{- 2 2 . 3 1 \%}$ & $\mathbf{- 1 6 . 5 5 \%}$ \\
\hline
\end{tabular}

Table 4. Index size and search speed

\subsubsection{Further reduction of index size}

Experiments are further conducted to reduce the index size by pruning on the two methods. Arcs on composite SCNs are discarded if their posterior probabilities are below a certain threshold. Further index size reduction is achieved with some performance loss, as shown in Figure 4. The top-left of the plot indicates more compact index and better performance. It is shown that the Lat.C method is the most efficient method. An interesting working point is when the two proposed method build indices in comparable size to the baseline system as shown in Table 5. A relative accuracy improvement of $13.11 \%$ is still achieved in this case.

\begin{tabular}{c||c|c|c|c}
\hline System & Index Size & FOM & rel.impr & Threshold \\
\hline Baseline & 1.56 & 56.05 & 0.00 & 0 \\
\hline Lat.C. & 1.58 & $\mathbf{6 3 . 4 0}$ & $\mathbf{1 3 . 1 1}$ & 0.002 \\
\hline CN.C & 1.63 & 63.27 & 12.88 & 0.002 \\
\hline
\end{tabular}

Table 5. Accuracy of combination methods when index size is comparable to baseline

\section{CONCLUSIONS}

In this paper, we explore efficient system combination methods for the SCN-based Chinese STD. Two new methods are proposed to combine the output of word/syllable systems into a single syllablelevel index. A relative accuracy improvement of $14.70 \%$ in FOM is achieved compared to the baseline system from 56.05 to 64.29 . Also by merging cross-system redundant information, it reduces the index size by $22.3 \%$ compared to the commonly adopted score combination method when achieves comparable accuracy. For future work,

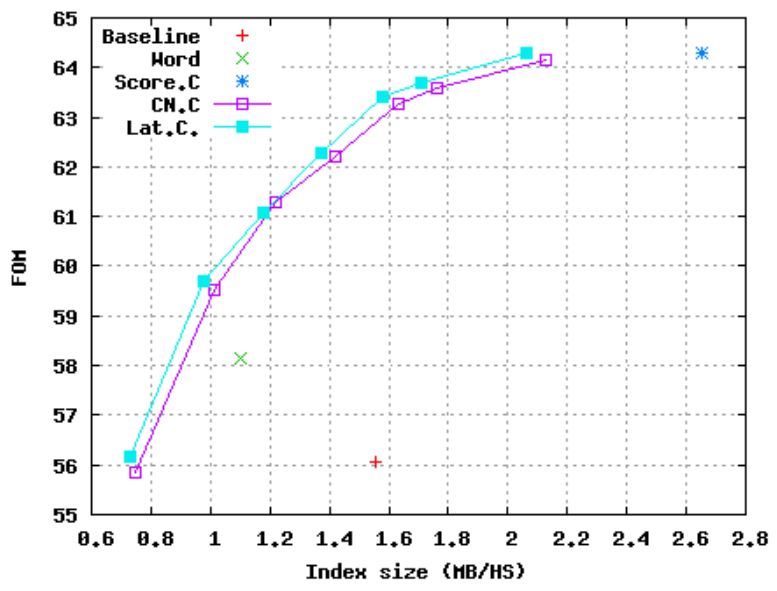

Fig. 4. Effect of pruning on the index size and accuracy

we would like to extend our work to systems of more units (character, syllable collocation etc.) and make further comparison with previous system combination methods.

\section{REFERENCES}

[1] D.Miller, M.Kleber, C.Kao, and O.Kimball. Rapid and accurate spoken term detection. Interspeech 2007, pages 314-317

[2] G.Evermann and P.Woodland. Posterior probability decoding, confidence estimation and system combination. The NIST 2000 Speech Transcription Workshop, 2000.

[3] http://www.nist.gov/speech/tests/std/2006/.

[4] J.Garofolo, G.Auzanne, and E.Voorhees. The TREC spoken document retrieval track : A success story. National Institute of Standards and Technology, 2000.

[5] J.Shao, Q.W.Zhao, P.Y.Zhang, and Y.Yan. A fast fuzzy keyword spotting algorithm based on syllable confusion network. Interspeech 2007, pages 2405-2408, August 2007.

[6] J.Shao, T.Li, Q.Zhang, Q.Zhao and Y.Yan. A one-pass realtime decoder using memory-effcient state network. IEICE Tran.INF \& SYST, E91-D(3):529-537, March 2008.

[7] L.Mangu, E.Brill, and A.Stolcke. Finding consensus in speech recognition: word error minimization and other applications of confusion networks. Computer Speech and Language, 14(4):373-400, January 2000.

[8] P.Yu and F.Seide. A hybrid word/phoneme-based approach for improved vocabulary-independent search in spontaneous speech. Interspeech 2004, pages 293-296, 2004.

[9] Y.Lee, K.Tanaka, and Y.Itoh. Combining multiple subword representatations for open-vocabulary spoken document retreival. ICASSP 2005, pages 505-508.

[10] S.Meng, P.Yu, F.Seide, and J.Liu. A study of lattice-based spoken term detection for Chinese spontaneous speech. ASRU 2007, pages 635-640, 2007.

[11] T.Hori, I.L.Hetherington, T.J.Hazen, and J.R.Glass. OpenVocabulary Spoken Utterance Retrieval using Confusion Networks. ICASSP 2007, pages 73-76, 2007.

[12] Y.Pan, H.Chang, B.Chen, and L.Lee. Subword-based Position Specific Posterior Lattices (S-PSPL) for Indexing Speech Information. Interspeech 2007, pages 318-321. 